\title{
miscellany
}

\section{Doctors need a listening ear}

Stress and burnout are well known in both hospital doctors and general practitioners. It has been reported that suicide rates are highest in anaesthetists, general practitioners and psychiatrists. Moreover, women doctors report higher levels of stress than men. In the London Deanery, psychological morbidity is highest in firstyear doctors, with around one-third having psychological distress. One survey estimated that $87 \%$ of doctors struggle into work when they are not fit to be there. Improving the health and wellbeing of the medical profession is slowly moving up the agendas of our professional organisations and the Department of Health, but what is out there to help the distressed doctor?

Doctors' SupportLine (DSL) is one of the few initiatives to tackle this problem. Doctors' SupportLine is a national telephone helpline for doctors where calls are answered by trained volunteer doctors The support line is anonymous and confidential to allow troubled doctors to take their first steps towards resolving issues that may be affecting their work and wellbeing. It is widely accepted that doctors are often unwilling to discuss their personal problems or to seek appropriate help. Not least this is because of fears that seeking help will be viewed as a sign of weakness, or that their careers will be damaged by colleagues being aware of their emotional problems and by the stigma of mental illness.

The helpline is unique because all calls are always answered by fellow doctors and callers remain anonymous. There is no doctor-patient relationship and counselling, but there is the opportunity to talk to a concerned and caring person who also comes from a medical background and therefore understands what it is really like to be a doctor. We offer active focused listening but not specific advice. Often to be listened to in a nonjudgemental and supportive way is help enough for the moment. If requested, we offer 'signposts' to other organisations which callers may find helpful. The Doctors' Supportline is completely independent and this should reassure callers that we have no other agenda than to be helpful and supportive. Like our callers, our trained volunteers are anonymous.

To date we have taken over 840 calls, which amount to 500 hours of helping our worried colleagues. We are a valued service within the medical profession and we feel we have contributed in some small way to improving the mental health of doctors.

If you have a problem and would like a confidential chat, we can be contacted on 08707650001 on Monday and Tuesday from 6 p.m. to 11 p.m., on Wednesday to Friday from 6 p.m. to 10 p.m. and on Sundays from 10 a.m. to 10 p.m.

Further information can be found on our website at http://www.

doctorssupport.org

Chris Williams Retired General Practitioner and Co-director of Doctors' SupportLine, 11 Percy Road Bournemouth BH5 1JF; tel: 01202398 014; email: doctist@ntlworld.com, Deirdre McLellan Project Manager , Doctors' SupportLine, Fulham Studios, 17-21 Wyfold Road, London SW6 6SE; tel: 02078355850; email: deirdre@doctorssupport.org 